\title{
Relationship between forward head posture and sacral slope angle
}

\author{
Salma M. Ahmed, Hamed M. Elkhozamy, Salwa F. Abdul Majeed and Alaa E. Abd EL \\ Hakim Balba
}

Department for Musculoskeletal Disorders and its Surgery, Faculty of Physical Therapy, Cairo University, Egypt.

Received: 10 Feb. 2019 / Accepted 16 Mar. 2019 / Publication date: 30 Dec. 2019

\begin{abstract}
Purpose: to investigate the relation between forward head posture and sacral slope angle in chronic neck pain patients. Methods: Forty patients of both genders 13 male and 27 female with chronic neck pain aged (18:30) were recruited from the outpatient physiotherapy clinic of Kaser elany medical school. Patients were divided into Group A (cranio-vertebral angel is $<46^{\circ} \mathrm{C}$ ) and Group B (cranio-vertebral angle is $>45^{\circ} \mathrm{C}$ ), the visual analogue scale, Oswestry disability index, electronic head posture instrument (EHPI), $x$ ray were used to measure neck pain, back function craniovertebral angle, sacral slope angle and pelvic incidence, respectively. Results: The Pearson correlation coefficient revealed that, Forward head posture was not significantly correlated neither with sacral slope angle $(\mathrm{r}=0.188 ; \mathrm{P}<0.05)$, nor with pelvic incidence $(\mathrm{r}=0.077$; $\mathrm{P}<0.05)$. Further cranio-vertebral angle) has no correlation with neck pain $(\mathrm{r}=0.077)$, and ODI $(\mathrm{r}=0.04)$. Conclusion: there is no correlation between forward head posture and pelvic parameters (pelvic incidence and sacral slope angle), these faulty head posture has no influence on neck pain and back function.
\end{abstract}

Keywords: Forward head posture, sacral slope angle, pelvic incidence, neck pain, back function

\section{Introduction}

Cone of economy represent human skeleton chain in perfect balance requiring a minimum of muscle activity in normal situations, in which the spinal segments with cephalic vertebra working as a pendulum to maintain horizontal gaze. (Ahmadi et al., 2015) (Hasegawa et al., 2017). Faulty posture due to different patterns of mechanical loading and asymmetrical stresses induce improper muscle contraction, which increase compressive loads on tissue (Sawyer, 2005; Dolphens et al., 2012). Thus posture mal-alignment leads to a decrease in the health-related quality of life.(Hasegawa et al., 2017) long period with forward head posture while doing work on laptop, in the activities of studying, and leisure on a computer, considered as important factors for developing pain at head the cervical region, the shoulder, and the lumbosacral spine (Fayyaz et al., 2017; Caromano et al., 2015). Forward head posture is the most common form of postural deviation in which it characterized as forward projecting of the head into the sagittal plane (Sajjadi et al., 2014). Hyper extension of upper cervical spine and flexion of lower cervical spine is a common form of forward head posture (Darnell, 1983) Chronic neck pain is a common problem associated with forward head posture (Gadotti, 2010; Mohankumar \& Yie, 2018), in which moderate positive correlation between FHP and duration of neck pain, however a negative relationship was found between intensity of neck pain and FHP with Prevalence approximately $37 \%$, out of which $58 \%$ were female and $42 \%$ were male(Wani et al., 2016). These faulty posture associated with dysafferentation (Morningstar et al., 2004). In addition development and persistence of many abnormalities including headaches (Cuadrado and Pareja 2006) neck pain syndrome (Yip et al., 2008; Subbarayalu and Ameer, 2017) abnormal scapular movement, and temporo-mandibular disorders (Song et al., 2015). The limited cervical range of motion has also been found in patients with tension-type headache (Fernández-de-las-Peñas et al., 2006; Quek et al., 2013)

Mal-alignment make the body to recruit compensatory mechanism to maintain horizontal eye gaze and line if gravity within base of support (Lamartina \& Berjano, 2014), compensation require changes in neuromuscular coordination in which the body rearrange its posture in adapting to a functional deficit to permit the least amount of pain and muscular effort expenditure (Filipović \&

Corresponding Author: Salma M. Ahmed, Department for musculoskeletal disorders and its surgery, Faculty of Physical Therapy, Cairo University, Egypt. 
Ciliga, 2010). such pelvo-ocular reflex in which anterior translation of pelvic secondary to forward head posture (Clark, Lucett, \& Sutton, 2011; Morningstar et al., 2005)further (Murtagh et al 2016) demonstrated that cervical sagittal mal-alignment can compensated with pelvic retroversion. (Wang et al., 2012). Hayden et al., (2018) suggested that the pelvis and spine co-exist as a dynamic linked system. When sagittal imbalance of spinal alignment occurs, compensatory mechanisms affect not only the spinal column but also the pelvis expressed by sacral slope (SS), indicating the position of the pelvis in the sagittal plane. compensatory mechanisms are efficient to limit the sagittal unbalance, they could result in adverse effects such as mechanical pain and compromise of neurological structures (Barrey et al., 2013) so it is better to evaluate the overall array of a body rather than determining the area of complain. (Choi et al., 2014)

The aim of this study was to investigate the relationship between the forward head posture and sacral slope angle in patient with chronic neck pain.

\section{Materials and Methods}

\section{Participants}

Forty patients of both genders 13 male and 27 female with chronic neck pain aged (18:30) were recruited from the outpatient physiotherapy clinic of Kaser elany medical school. Patients were divided into Group A (cranio-vertebral angel is $<46^{\circ}$ ) and Group B (cranio-vertebral angle is $>45^{\circ}$ ), All the patients approved the study protocol and provided an informed consent for their participation. Patients were excluded from the study if they had a history of cervical fracture, trauma, scoliosis, bone cancer, spasmodic torticollis, neurological disorder or cervical radiculopathy.

Hearing impairment, pregnant female, temporo-mandibular surgery, body mass index above 30 , systemic disorder were also excluded from the study.

\section{Procedure:}

\section{Pain assessment}

Pain was assessed by Visual Analogue Scale (VAS) which is the optimal tool with has high reliability (CC=0.94) ( Hawker et al., 2011).

\section{Back function assessment}

Back function was assessed by Oswestry Disability Index (ODI): It is instrument with high internal consistency $(=0.71-0.8)$ and test-retest reliability (intra-class correlation coefficient 0.847 .), It is a self-reported questionnaire comprised of 10 questions which asks patients about their ability to manage everyday life and covers intensity of pain, lifting, the ability to care for oneself, ability to walk, the ability to sit, sexual function, ability to stand, social life, sleep quality, and ability to travel. Answers are then scored on a 0-5 scale, zero meaning no disability 5 representing the greatest disability (Fairbank and Pynsent, 2000; Smeets., 2011).

\section{Head posture measurement}

CV angle was measured by the EHPI, which was composed of an electronic angle finder attached with metal ruler supported on camera stand, The assessor first located the spinous process of $\mathrm{C} 7$ by asking the subject to flex and extend the neck. The $\mathrm{C} 7$ spinous process is more prominent, whereas the $\mathrm{C} 6$ spinous process is absent in palpation when the neck is extended. The $\mathrm{C} 7$ spinous process was then marked by a small flag to ensure the correct location and consistency of the bony landmark. Another small flag fixed at the tragus of dominant hand of patient, During the assessment, the patient was required to stand in a relaxed posture .The patient was then instructed to flex and extend his or her head three times and then rest the head in a comfortable neutral position (Yip et al., 2008). The assessor performed the assessment in sagittal view of dominant side of patient, the assessor aligned the axis of the camera stand with the $\mathrm{C} 7$ spinous process, The EHPI was put on the standardized marking on the floor, and the camera stand was adjusted to the position until the bubble of the horizontal indicator and the central marking overlapped. The distance from the participant's shoulder tip to the center of the tripod stand was standardized to $0.3 \mathrm{~m}$ while the distance between the assessor's eyes and the tripod stand was $0.5 \mathrm{~m}$ because this was the longest distance that the assessor 
could reach A virtual line was drawn between the two pin markers from midpoints of the tragus to C7. The therapist adjusted the EHPI until the two indicator lines were aligned with the markers. The reading from the Angle Finder represented the CV angle (Lau et al., 2009).

\section{Measurement of sacral slope angle:}

Lateral radiograph of the pelvic was taken from a constant distance between the subject and the radiographic source, nearly $(100 \mathrm{~cm})$. The subject stood in a comfortable position, with the knees fully extended and the arms flexed forward to $45^{\circ}$ and resting on supports. The radiograph was centered on the twelfth thoracic vertebra and was made during inhalation. The measurements were made using Surgimap (Nemaris Inc, New York, NY) with excellent reliability (ICC >0.9) (Lafage et al., 2015) Spino-pelvic parameters are automatically generated by the software based on the demarcated landmarks and splines.

- Sacral slope is the angle between the horizontal line and the cranial sacral end-plate tangent (Legaye et al., 1998).

-Pelvic incidence: is the angle between the perpendicular line to the sacral plate at its midpoint and the line connecting the point to the middle axis of the femoral heads (Legaye et al., 1998).

\section{Statistical analysis}

A Pearson correlation coefficient was applied to study the strength and direction of a linear relationship between variables such as Cranio-vertebral angle, visual analogue scale, sacral slope angle pelvic incidence, Oswestry disability index (ODI), Statistical analysis was conducted using SPSS for windows, version 23 (SPSS, Inc., Chicago, IL). Further, one way MANOVA was conducted to compare all dependent variables between both groups, the alpha level was set at 0.05 .

Independent $t$ test was used to determine descriptive statistics analysis for general characteristic of subjects values (age, body mass, height, and BMI) for the both tested group, further Chi square test was used to determine differences in sex between both groups.

\section{Results}

\section{Morphological characteristics of participants in the study.}

The Pearson correlation coefficient revealed that, Forward head posture was significantly correlated neither with sacral slope angle $(\mathrm{r}=0.188 ; \mathrm{P}<0.05)$, nor with pelvic incidence $(\mathrm{r}=0.077 ; \mathrm{P}$ $<0.05)$. Further (CVA) has no correlation with neck pain $(\mathrm{r}=0.077)$, and ODI $(\mathrm{r}=0.04)$

Statistical analysis using one way MANOVA was conducted to compare all variables between both groups

Table 1: Descriptive Statistics of Participants age, weight, height, BMI, and gender.

\begin{tabular}{|c|c|c|c|c|c|}
\hline & $\begin{array}{c}\text { Group A } \\
(\mathbf{N}=22)\end{array}$ & $\begin{array}{c}\text { Group B } \\
(\mathrm{N}=18)\end{array}$ & t-value & $P$-value & $\begin{array}{c}\text { Level of } \\
\text { significant }\end{array}$ \\
\hline Age (years) & $23.09 \pm 3.37$ & $23.44 \pm 2.74$ & -0.357 & 0.723 & N.S \\
\hline Bodymass (kg) & $63.8 \pm 12.05$ & $66.45 \pm 9.15$ & -0.767 & 0.448 & N.S \\
\hline Height $(\mathrm{cm})$ & $162.1 \pm 8.82$ & $168.61 \pm 8.07$ & -2.411 & 0.05 & N.S \\
\hline BMI (kg/m2) & $24.16 \pm 3.55$ & $23.33 \pm 2.63$ & 0.828 & 0.413 & N.S \\
\hline \multicolumn{6}{|c|}{ Sex distribution N (\%) } \\
\hline & Group A & Group B & $\mathbf{X} 2$ & P-value & $\begin{array}{c}\text { Level of } \\
\text { significant }\end{array}$ \\
\hline $\begin{array}{c}\text { Female } \\
\text { Male }\end{array}$ & $\begin{array}{c}16(72.7 \%) \\
6(27.3 \%)\end{array}$ & $\begin{array}{c}11(61.2 \%) \\
7(38.8 \%)\end{array}$ & 0.609 & 0.509 & N.S \\
\hline
\end{tabular}

*Significant at alpha level $<0.05$

\section{1-Sacral angle between both groups}

The mean \pm SD values of sacral angle in the "group A" and "group B" were $37.7 \pm 6.45$ and $37.2 \pm 8.2$ respectively. Multiple pairwise comparison tests (Post hoc tests) revealed that there was no significant difference in sacral angle in group B in compared with group A with $(\mathrm{F}=0.047, \mathrm{p}=0.829)$. 


\section{2-Pelvic incidence between both groups}

The mean \pm SD values of pelvic incidence in the "group A" and "group B" were $52.4 \pm 8.77$ and $49.01 \pm 8.56$ respectively. Multiple pairwise comparison tests (Post hoc tests) revealed that there was no significant difference in pelvic index in group B in compared with group A with $(\mathrm{F}=1.511, \mathrm{p}=$ $0.227)$.

\section{3-VAS between both groups}

The mean \pm SD values of VAS in the "group A" and "group B" were $4.78 \pm 1.54$ and $4.83 \pm 1.98$ respectively. Multiple pairwise comparison tests (Post hoc tests) revealed that there was no significant difference in VAS in group $B$ in compared with group A with $(F=0.01, p=0.921)$.

\section{4-Oswestry disability index between both groups}

The mean $\pm \mathrm{SD}$ values of Oswestry disability index in the "group A" and "group B" were $14.46 \pm 8.24$ and 14.63 \pm 7.74 respectively. Multiple pairwise comparison tests (Post hoc tests) revealed that there was no significant difference in Oswestry disability index in group B in compared with group A with $(\mathrm{F}=0.004, \mathrm{p}=0.948)$.

\section{Discussion}

\section{Forward head posture and sacral slope angle}

This study was conducted to investigate the relationship between forward head posture and sacral slope angle, this study utilized a non-invasive instrument with high reliability (Lau et al., 2009), EHPI was validated by (Lau et al., 2010) to measure forward head posture, further sacral slope angle was measured by $\mathrm{x}$ ray. To our knowledge, no previous studies have investigated the relation between forward head posture and sacral slope angel. The results of this study demonstrated that Forward head posture has no correlation with sacral slope angle $(\mathrm{r}=0.188 ; \mathrm{P}<0.05)$, and pelvic incidence $(\mathrm{r}=0.077 ; \mathrm{P}$ $<0.05)$. The mean sacral angle, cranio-vertebral angle and pelvic incidence was $(36.51 \pm 9.32) \&$ (46.6 \pm 5.02$),(49.6 \pm 11.88)$ respectively.

The results of this study was consistent with Endo et al. (2016) finding who evaluate the association between cervical sagittal alignment and thoracic/lumbo pelvic sagittal alignment and suggested that, the cervical sagittal vertical axis did not correlate with lumbar lordosis, sacral slope, or pelvic tilt further ,Pelvic incidence correlated with the sacral slope and lumbar lordosis, but not with thoracic kyphosis or cervical alignment.

This finding is in line with finding of study was conducted to investigate the effect of forward head posture on spinal curvature on healthy subjects by Talati et al. (2018) in which they noted that there is no significant correlation between forward head posture, thoracic kyphosis, and lumbar lordosis in normal adults aged 18-35 years, further Boulet et al., (2016) who investigated Relationships between each part of the spinal curves, noted that no significant correlation between cervical lordosis angle(between the T4 and C3 vertebra) and thoracic kyphosis and lumbar lordosis.

According to Singla \& Veqa, (2017) mal-alignment of head posture ,alter position line of gravity, thus become anterior to $\mathrm{c} 7$ in forward head posture, the body recruit the compensatory mechanism to relocate line of gravity within base of support, but (Roussouly et al., 2006) noted a significant correlation between the position of the gravity line and the pelvic tilt $(\mathrm{R}=0.746, \mathrm{P}<0.001)$, but no correlation between the position of the gravity line and the sacral slope $(\mathrm{R}=0.058, \mathrm{P}<0.473)$.

It is possible that the individual exhibit different compensatory mechanisms to restore horizontal eye gaze and line of gravity within base of support thus, compensatory mechanism may be regional at adjacent segment and adjacent curve or may be global, further according to (Lamartina \& Berjano, 2014) findings who classified compensatory mechanism into two categories: compensated imbalance, in which compensation can occur by spinal or non-spinal segments consequently the body uses muscle force to improve alignment with a resulting spinal shape that restores the gravity line position and horizontal gaze .In this stage, the individual is able to meet these alignment goals, but at the price of increased muscle activity, in case of Increasing deformity or inadequate muscle strength or endurance in the Individual can make compensatory mechanisms to fail This situation is defined as 
uncompensated imbalance (Gangnet et al., 2003) also found large variability of the spine configuration with respect to the gravity line.

On contrary Polansky et al. (1996) investigated the influence of different sitting posture on cervical and lumbar posture and concluded that, correlation between Lumbar curve and total angle of cervical spine(position of head relative to cervical base) are strongly correlated, as the position of lumbar orient toward extension, The cervical spine tend to move toward flexion, in addition the result of Choi et al. (2014) revealed that Lumbar lordosis is largely affected by sacral slope angle.

further, Narrative review of Ames et al., (2013) concluded that CL depends on both TK and LL ,CL can be considered an adaptive spinal segment in which it changes relative to the other spinal segments to attempt to maintain the head over the pelvis and maintain horizontal gaze.

However Ames et al. (2013) stated that A large Pelvic incidence requires a large sacral slope angle , large Lumbar lordosis. An increase of Lumbar Lordosis is correlated with an increased Thoracic kyphosis, which is correlated with an increased Cervical Lordosis, thus the difference between the results could reflect structural differences that our study didn't take it into account.

\section{Neck pain and forward head posture}

The result of these study revealed that there is no significant differences between forward head posture and VAS in patients with chronic neck pain.

Our finding is consistent with systematic review ,conducted by Silva et al. (2010) that compared Head Posture between participants with NP and asymptomatic participants, which could not find relation between forward head posture and neck pain. Also the finding of these study is in line with (Ghamkhar \& Hossein, 2018) finding, who investigate the relation between FHP in Chronic Neck pain patients and asymptomatic participants in which the mean VAS of these study was $(3.95 \pm 2.15)$ and the mean VAS of our study was(4.07 \pm 2.19$)$, which concluded, that the cranio-vertebral angle (defining FHP) was not different between the chronic neck pain patient and the asymptomatic groups.

It is possible that no correlation exist due to sub-classifications of neck pain in which etiology, pathophysiology, location and duration of symptoms and control of these factors was lacked in these study. Also, lack of association between Neck Pain and cervico-thoracic posture was suggested by Straker et al. (2009) which concluded that posture may have only a small influence on neck pain.

In addition a study of Yip et al. (2008) who measured the CV angle by using the Head Posture Spinal Curvature Instrument (HPSCI) that has high degree of reliability in chronic neck pain patients to investigate the relationship between head posture with pain and disability in patients with neck pain and concluded that The correlation between $\mathrm{CV}$ angle and neck pain were only moderate at best.

On other hand Haughie et al. (1995) reported that craino-vertebral angle decrease in chronic neck patient,when investigated the relationship of forward head posture and cervical backward bending to neck pain in which the cranio-vertebral angle was measured by CROM and the mean age of the subjects in the case group was 46 years old and have pain in 4 area or more, the control group was 44 years of age with pain in three areas or less were considered the control group. The mean age in our study was $23.25 \pm 3.07$. further according to Raine \& Twomey, (1997) finding the relation between sagittal C7-tragus angle and age was statistically significant, indicating that subjects had a more forward-placed head with increasing age.

In addition a study of Mohankumar \& Yie, (2018) who assessed head and neck posture in young adults with chronic neck pain by measuring cranio-vertebral angle by Photographic method with patients at the same age of our study range from (20:30) and mean of cranio-vertebral angle was 53.2 in neck pain group which numerical Rating Scale (NRS) score for pain from 3 to 7 and 50.5 in healthy subjects group he concluded that forward head posture predisposes people to suffer neck pain. The difference between result of our study and the result of these study could explained by Yip et al., 2008) study who concluded that the association between forward head posture and level of neck pain might reveal about $10-15 \%$ (if it were a causative relationship), but there are many factor associated with neck pain for example, osteoarthritis changes, repetitive strain, overuse syndromes and psychological factors that can contribute to the level of neck pain and disabilities.

In addition the mean of cranio-vertebral angle of our study is $46.6 \pm 5.02$ which is smaller than the mean of these study, the difference between these study and ours could be explained faulty posture might increase loading to the non-contractile structures and abnormal stress on the posterior cervical structures and cause myofascial pain. 
So prolonged being in faulty posture make the individuals have adapted to postural changes to permit the least amount of pain and muscular effort expenditure (Christie et al., 1995). In addition to (De-La-Llave-Rincón et al., (2009 who noted that the change of head posture may be a consequence ( an antalgic posture in trying to reduce pain, rather than a possible etiologic factor). However, to date the association between forward head posture and neck pain is controversial.

\section{Forward head posture and Oswestry disability index}

In these study Pearson correlation coefficient (r) between mean value of Cranio-vertebral angle and Oswestry disability index for back function was 0.04 which means there was no correlation $(\mathrm{p}=0.808)$.thus, change in the Cranio-vertebral angle is not consistent with change in Oswestry disability index

The finding of present study is in line with Christensen \& Hartvigsen, (2008) who conducted a systematic critical review of epidemiological studies published between 1942 and 2008 does not support any association between sagittal spinal curves and health, including spinal pain and function. Similarly systematic review has been conducted by Dieck et al., (1985) who didn't find any relation between posture and pain

In addition, Endo et al. (2016) results who investigated relationship among cervical, thoracic, and lumbo-pelvic sagittal alignment in healthy adults and concluded that cervical sagittal vertical axis did not correlate with lumbar lordosis sacral slope, or pelvic tilt. In which increase lumbar lordosis and pelvic tilt associated with back dysfunction and pain. (Day, Smidt, \& Lehmann, 1984).

In contrast Christie et al. (1995) investigated the Postural Aberrations in Low Back Pain and noted that faulty postural changes alignment with respect to the line of gravity that may lead to other adaptive postural changes that induce pain and dysfunction.

Further, study of Cădar \& Pop, (2015) who investigate the importance of cervical spine posture rehabilitation in treatment of low back pain concluded that biomechanical changes due to forward head lead to modification of the body gravity-center, which will overuse the lumbar spine. In these conditions the lumbar spine won't be able to balance the body on the pelvis girdle and lower limbs consequently, degeneration mainly of the spine structures, but also of the other joints, muscular imbalances, inflammations and faulty movement patterns which compromise the whole body dynamic.

The difference between findings of the studies could explained according to Womersley \& May, (2006) study who found that minimal level of spinal muscle activity have been noted in relaxed sitting postures in young adult, and pain due to extreme spinal positions is provoked by mechanical load on articular and periarticular structures rather than sustained muscular activity Thus, the mechanism of pain stimulation in postural backache could be mechanical tension stress on soft tissues without overt tissue damage. Once the individual leaves that posture, the tension is released and the discomfort abates without more serious sequelae, thus persistent maintained poor posture throughout life may has implications on back ache on older population and contribute to loss of movement and function.

\section{Conclusion}

On the basis of the present results it is possible to conclude that, there was no correlation between forward head posture and sacral slope angle, The CV angle has no correlation with VAS in neck pain patient, further head posture doesn't influence back function.

\section{References}

Ahmadi, A., N. Maroufi and J. Sarrafzadeh, 2016. Evaluation of forward head posture in sitting and standing positions. European Spine Journal, 25(11), 3577-3582.

Ames, C. P., B. Blondel, J. K. Scheer, F.J. Schwab, J.C. Le Huec, E. M Massicotte and J. S. Smith, 2013. Cervical radiographical alignment: comprehensive assessment techniques and potential importance in cervical myelopathy. Spine, 38(22S), S149-S160.

Barrey, C., P. Roussouly, J.C. Le Huec, G. D’Acunzi and G. Perrin, 2013. Compensatory mechanisms contributing to keep the sagittal balance of the spine. European Spine Journal, 22(6), 834-841. 
Boulet, S., E. Boudot and N. Houel, 2016. Relationships between each part of the spinal curves and upright posture using multiple stepwise linear regression analysis. Journal of Biomechanics, 49(7): 1149-1155.

Cădar, I. D., and L.V. Pop, 2015. Correlations between cervical spine posture and low back pain. Human and Veterinary Medicine, 7(3): 178-181.

Caromano, F. A., C. A. P. de Amorim, C. de Fátima Rebelo, A.M. Contesini, F.M. Fávero, J.R.C. Frutuoso and M.C. Voos, 2015. Permanência prolongada na postura sentada e desconforto físico em estudantes universitários. Acta físiátrica, 22(4): 176-180.

Choi, S., M. Lee and B. Kwon, 2014. A study on difference and importance of sacral slope and pelvic sacral angle that affect lumbar curvature. Technology and Health Care, 22(3), 467-472.

Christensen, S.T. and J. Hartvigsen, 2008. Spinal curves and health: a systematic critical review of the epidemiological literature dealing with associations between sagittal spinal curves and health. Journal of Manipulative and Physiological Therapeutics, 31(9):690-714.

Christie, H. J., S. Kumar and S.A. Warren, 1995. Postural aberrations in low back pain. Archives of Physical Medicine and Rehabilitation, 76(3): 218-224.

Clark, M.A., S. Lucett and R.J. Corn, 2008. NASM essentials of personal fitness training. Lippincott Williams \& Wilkins.

Darnell, M.W., 1983. A proposed chronology of events for forward head posture. Journal of Craniomandibular Practice, 1(4): 49-54.

Day, J., G. Smidt and T. Lehmann, 1984. Effect of Pelvic Tilt on Standing Posture. Physical Therapy, 64(4): 510-516.

De-La-Llave-Rincón, A.I., C. Fernández-De-Las-PeÑas, D. Palacios-CeÑa and J.A. Cleland, 2009. Increased forward head posture and restricted cervical range of motion in patients with carpal tunnel syndrome. Journal of Orthopaedic \& Sports Physical Therapy, 39(9): 658-664.

Dieck, G.S., J.L. Kelsey, V.K. Goel, M.M. Panjabi, S.D. Walter and M.H. Laprade, 1985. An epidemiologic study of the relationship between postural asymmetry in the teen years and subsequent back and neck pain. Spine, 10(10): 872-877.

Dolphens, M., B. Cagnie, P. Coorevits, G. Vanderstraeten, G. Cardon, R. D'hooge and L. Danneels, 2012. Sagittal standing posture and its association with spinal pain: a school-based epidemiological study of 1196 Flemish adolescents before age at peak height velocity. Spine, 37(19):1657-1666.

Endo, K., H. Suzuki, Y. Sawaji, H. Nishimura, M. Yorifuji, K. Murata and K. Yamamoto, 2016. Relationship among cervical, thoracic, and lumbopelvic sagittal alignment in healthy adults. Journal of Orthopaedic Surgery, 24(1), 92-96.

Fairbank, J. C. and P.B. Pynsent, 2000. The Oswestry disability index. Spine, 25(22), 2940-2953.

Fayyaz, A., F. Shoukat and S.A. Gilani, 2017. Contribution of Bad Posture towards Development of 'Adolescent Scoliosis' among University Students. Journal of Fatima Jinnah Medical University, 11(3): 46-50.

Fernández-de-Las-Peñas, C., M. L. Cuadrado and J.A. Pareja, 2006. Myofascial trigger points, neck mobility and forward head posture in unilateral migraine. Cephalalgia, 26(9):1061-1070.

Fernández-de-las-Peñas, C., C. Alonso-Blanco, M. L. Cuadrado, R.D. Gerwin and J. A. Pareja, 2006. Trigger points in the suboccipital muscles and forward head posture in tension-type headache. Headache: The Journal of Head and Face Pain, 46(3), 454-460.

Filipović, V. and D. Ciliga, 2010. Postural adaptation of idiopathic adolescent scolioses (IAS). International Journal of Fundamental and Applied Kinesiology, 42(1):16-27.

Gadotti, I. C. 2010. Measurement properties of the sagittal craniocervical posture photogrammetry.

Gangnet, N., V. Pomero, R. Dumas, W. Skalli and J.M. Vital, 2003. Variability of the spine and pelvis location with respect to the gravity line: a three-dimensional stereoradiographic study using a force platform. Surgical and Radiologic Anatomy, 25(5-6), 424-433.

Ghamkhar, L., and A.H. Kahlaee, 2018. Is forward head posture relevant to cervical muscles performance and neck pain? A case-control study. Brazilian Journal of Physical Therapy, 23(4):346-354.

Hawker, G. A., S. Mian, T. Kendzerska and M. French, 2011. Measures of adult pain: Visual analog scale for pain (vas pain), numeric rating scale for pain (nrs pain), mcgill pain questionnaire (mpq), short-form mcgill pain questionnaire (sf-mpq), chronic pain grade scale (cpgs), short 
form-36 bodily pain scale (sf-36 bps), and measure of intermittent and constant osteoarthritis pain (icoap). Arthritis care \& research, 63(S11), S240-S252.

Hasegawa, K., M. Okamoto, S. Hatsushikano, H. Shimoda, M. Ono, T. Homma and K. Watanabe, 2017. Standing sagittal alignment of the whole axial skeleton with reference to the gravity line in humans. Journal of anatomy, 230(5): 619-630.

Haughie, L.J., I.M. Fiebert and K.E. Roach, 1995. Relationship of forward head posture and cervical backward bending to neck pain. Journal of Manual \& Manipulative Therapy, 3(3): 91-97.

Hayden, A.M., A.M. Hayes, J. L. Brechbuhler, H. Israel and H. M. Place, 2018. The effect of pelvic motion on spinopelvic parameters. The Spine Journal, 18(1), 173-178.

Lafage, R., E. Ferrero, J. Henry, V. Challier, B. Diebo and Liabaud, B. et al. 2015. Validation of a new computer-assisted tool to measure spino-pelvic parameters. The Spine Journal, 15(12):2493-2502.

Lamartina, C., and P. Berjano, 2014. Classification of sagittal imbalance based on spinal alignment and compensatory mechanisms. European Spine Journal, 23(6): 1177-1189.

Lau, H.M.C., T.T.W. Chiu and T.H. Lam, 2009. Clinical measurement of craniovertebral angle by electronic head posture instrument: a test of reliability and validity. Manual therapy, 14(4): 363368.

Lau, H.M.C., T.T.W. Chiu and T.H. Lam, 2010. Measurement of craniovertebral angle with Electronic Head Posture Instrument: Criterion validity. Journal of Rehabilitation Research and Development, 47(9): 911-918.

Legaye, J., G. Duval-Beaupere, J. Hecquet and C. Marty, 1998. Pelvic incidence: a fundamental pelvic parameter for three-dimensional regulation of spinal sagittal curves. European Spine Journal, 7(2): 99-103.

Mohankumar, P., and L.W. Yie, 2018. Head and Neck Posture in Young Adults with Chronic Neck Pain, International Journal of Recent Advances in Multidisciplinary Research 04(11).2946-2951

Morningstar, M.W., B.R. Pettibon, H. Schlappi, M. Schlappi and T.V. Ireland, 2005. Reflex control of the spine and posture: a review of the literature from a chiropractic perspective. Chiropractic \& Osteopathy, 13(1): 16.

Morningstar, M.W., M.N. Strauchman and G. Gilmour, 2004. Adolescent idiopathic scoliosis treatment using Pettibon corrective procedures: a case report. Journal of chiropractic medicine, 3(3): 96-103.

Murtagh, R.D., R.M. Quencer and J. Uribe, 2016. Pelvic evaluation in thoracolumbar corrective spine surgery: how I do it. Radiology, 278(3): 646-656.

Black, K.M., P. McClure and M. Polansky, 1996. The influence of different sitting positions on cervical and lumbar posture. Spine, 21(1): 65-70.

Quek, J., Y.H. Pua, R.A. Clark and A.L. Bryant, 2013. Effects of thoracic kyphosis and forward head posture on cervical range of motion in older adults. Manual therapy, 18(1): 65-71.

Raine, S. and L.T. Twomey, 1997. Head and shoulder posture variations in 160 asymptomatic women and men. Archives of physical medicine and rehabilitation, 78(11): 1215-1223.

Roussouly, P., S. Gollogly, O. Noseda, E. Berthonnaud and J. Dimnet, 2006. The vertical projection of the sum of the ground reactive forces of a standing patient is not the same as the C7 plumb line: a radiographic study of the sagittal alignment of 153 asymptomatic volunteers. Spine, 31(11): E320-E325.

Sajjadi, E., G.R. Olyaei, S. Talebian, M.R. Hadian and S. Jalaie, 2014. The effect of forward head posture on cervical joint position sense. Journal of Paramedical Sciences (JPS), 5(4): 20084978.

Sawyer, Q. L., 1993. Effects of forward head rounded shoulder posture on shoulder girdle flexibility, range of motion, and strength.

Silva, A.G., P. Sharples and M.I. Johnson, 2010. Studies comparing surrogate measures for head posture in individuals with and without neck pain. Physical Therapy Reviews, 15(1): 12-22.

Singla, D., and Z. Veqar, 2017. Association between forward head, rounded shoulders, and increased thoracic kyphosis: a review of the literature. Journal of chiropractic medicine, 16(3): 220-229.

Smeets, R., A. Köke, C.W. Lin, M. Ferreira and C. Demoulin, 2011. Measures of function in low back pain/disorders: Low Back Pain Rating Scale (LBPRS), Oswestry Disability Index (ODI), Progressive Isoinertial Lifting Evaluation (PILE), Quebec Back Pain Disability Scale (QBPDS), 
and Roland-Morris Disability Questionnaire (RDQ). Arthritis Care \& Research, 63(S11), S158S173.

Song, J.I., S.Y. Kang, J.H. Park, H.S. Cynn and H. S. Jeon, 2015. Influence of Forward Head Posture on Electromyography Activity of Hyoid Muscles during Mouth Opening. Physical Therapy Korea, 22(1): 103-109.

Straker, L. M., P. B. O'Sullivan, A. J. Smith and M.C. Perry, 2009. Relationships between prolonged neck/shoulder pain and sitting spinal posture in male and female adolescents. Manual Therapy, 14(3), 321-329.

Subbarayalu, A.V. and M.A. Ameer, 2017. Relationships among head posture, pain intensity, disability and deep cervical flexor muscle performance in subjects with postural neck pain. Journal of Taibah University Medical Sciences, 12(6): 541-547.

Talati, D., G. Varadhrajulu and M. Malwade, 2018. The effect of forward head posture on spinal curvatures in healthy subjects, 5(1): 3-6.

Wang, H.J., H. Giambini, W.J. Zhang, G.H. Ye, C. Zhao K. N. An and Q. L. Zou, 2012. A modified sagittal spine postural classification and its relationship to deformities and spinal mobility in a Chinese osteoporotic population. PLoS One, 7(6): e38560.

Wani, Subrat, S., P. Ostwal and R. Quazi, 2016. Prevalence of Anterior Head Translation in Patients with Neck Pain . International Journal of Current Medical and Applied Science, 9(2): 78-83.

Womersley, L., and S. May, 2006. Sitting posture of subjects with postural backache. Journal of Manipulative and Physiological Therapeutics, 29(3): 213-218.

Yip, C.H.T., T.T.W. Chiu and A.T.K. Poon, 2008. The relationship between head posture and severity and disability of patients with neck pain. Manual therapy, 13(2):148-154. 